\title{
Cyclin D1 expression in peripheral T-cell lymphomas
}

Joo Y Song ${ }^{1}$, Liping Song ${ }^{1}$, Alex F Herrera ${ }^{2}$, Girish Venkataraman ${ }^{3}$, Joyce L Murata-Collins ${ }^{1}$, Victoria H Bedell ${ }^{1}$, Yuan Yuan Chen ${ }^{1}$, Young S Kim ${ }^{1}$, Reda Tadros ${ }^{4}$, Bharat N Nathwani ${ }^{1}$, Dennis D Weisenburger ${ }^{1}$ and Andrew L Feldman ${ }^{5}$

${ }^{1}$ Department of Pathology, City of Hope National Medical Center, Duarte, CA, USA; ${ }^{2}$ Department of Hematology and Hematopoietic Cell Transplantation, City of Hope National Medical Center, Duarte, CA, USA; ${ }^{3}$ Department of Pathology, University of Chicago, Chicago, IL, USA; ${ }^{4}$ Department of Pathology, Chino Valley Medical Center, Chino, CA, USA and ${ }^{5}$ Department of Laboratory Medicine and Pathology, Mayo Clinic, Rochester, MN, USA

\begin{abstract}
Cyclin D1 is an important regulator of the cell cycle and overexpression of this protein by immunohistochemistry is characteristically seen in mantle cell lymphoma and other B-cell neoplasms. However, little is known about the expression of this protein in T-cell lymphomas. Cyclin-dependent kinase pathway inhibitors are in development, therefore identifying cyclin D1-positive T-cell lymphomas may provide a therapeutic target in a disease where novel treatments are urgently needed. We collected 200 peripheral T-cell lymphomas from three institutions including the following types of cases: 34 anaplastic large cell lymphoma, ALK+, 44 anaplastic large cell lymphoma, ALK negative, 68 peripheral T-cell lymphomas, not otherwise specified, 24 angioimmunoblastic T-cell lymphomas, 7 extranodal NK/T-cell lymphomas, 4 enteropathy associated T-cell lymphomas, 3 hepatosplenic T-cell lymphomas, 12 cutaneous T-cell lymphomas, and 4 large granular lymphocytic leukemias. Immunohistochemical stains for cyclin D1 protein (SP4 clone) were performed on paraffin-embedded tissue. In a subset of cases, IGH/CCND1 fluorescence in situ hybridization analysis was also performed. Cyclin D1 staining was predominantly seen in anaplastic large cell lymphoma, including 8 of 34 cases with ALK+ anaplastic large cell lymphoma (24\%), and 3 of 44 cases of ALK-negative (7\%) anaplastic large cell lymphoma. Three cases of peripheral T-cell lymphoma, not otherwise specified, were also positive $(3 / 68,4 \%)$. All other T-cell lymphomas were negative for cyclin D1. In four of the cyclin D1-positive T-cell lymphomas by immunohistochemistry, fluorescence in situ hybridization analysis was negative for IGH/CCND1 translocation or extra copies of the CCND1 gene. Cyclin D1 overexpression by immunohistochemistry is not limited to B-cell lymphomas and is also observed in some peripheral T-cell lymphomas, particularly in anaplastic large cell lymphoma, ALK+. Cyclin D1 expression was not associated with extra copies or translocation of the CCND1 gene. Cyclin D1 overexpression may be the result of a post-translational phenomenon and may represent a potential therapeutic target using agents that target the cyclin-dependent kinase pathway.

Modern Pathology (2016) 29, 1306-1312; doi:10.1038/modpathol.2016.136; published online 29 July 2016
\end{abstract}

The cyclin proteins are important in the regulation of the cell cycle by activating cyclin-dependent kinases at specific stages of the cell cycle and thus promoting DNA replication and cell division. The cyclin D class of proteins are closely related to the G1 cyclins and activate cyclin-dependent kinase 4 and cyclindependent kinase $6 .{ }^{1}$ Mitogenic stimuli activate the

Correspondence: Dr JY Song, MD, Department of Pathology, City of Hope National Medical Center, 1500 E. Duarte Road, Duarte, CA 91010, USA.

E-mail: josong@coh.org

Received 11 April 2016; revised 2 June 2016; accepted 2 June 2016; published online 29 July 2016 tyrosine kinase receptor, which triggers a signaling cascade, resulting in upregulation of cyclin D1.,3 The deregulation of cyclin-dependent kinases can lead to cancer by causing proliferation that is independent of the checkpoints and regulators. ${ }^{1,4}$ Overexpression of cyclin D1 protein is implicated in many tumors such as mantle cell lymphoma, nonsmall cell lung cancer, plasma cell myeloma, hairy cell leukemia, as well as breast and esophageal cancers. ${ }^{1}$ Genomic alterations such as the $\mathrm{t}(11 ; 14)$ (q13;q32), which juxtaposes the cyclin D1-encoding gene (CCND1) to the immunoglobulin heavy chain gene, are characteristic for mantle cell lymphoma ${ }^{5}$ but is also seen in plasma cell myeloma. ${ }^{6}$ 
Table 1 Peripheral T-cell lymphomas immunostaining pattern

\begin{tabular}{|c|c|c|c|c|}
\hline \multirow{2}{*}{ T-cell lymphoma subtype } & \multicolumn{4}{|c|}{ No. $(\%)$} \\
\hline & Cyclin D1 & Cyclin D2 & Cyclin D3 & pStat3 \\
\hline Anaplastic large cell lymphoma, ALK+ & $8 / 34(24 \%)$ & $10 / 12(83 \%)$ & $12 / 12(100 \%)$ & $13 / 13(100 \%)$ \\
\hline Anaplastic large cell lymphoma, ALK- & $3 / 44(7 \%)$ & $7 / 15(47 \%)$ & $5 / 15(33 \%)$ & $6 / 16(38 \%)$ \\
\hline Peripheral T-cell lymphoma, NOS & $3 / 68(4 \%)$ & & & \\
\hline Angioimmunoblastic T-cell lymphoma & $0 / 24(0 \%)$ & & & \\
\hline Extranodal NK/T-cell lymphoma, nasal type & $0 / 7(0 \%)$ & & & \\
\hline Enteropathy associated T-cell lymphoma & $0 / 4(0 \%)$ & & & \\
\hline Hepatosplenic T-cell lymphoma & $0 / 3(0 \%)$ & & & \\
\hline Cutaneous anaplastic large cell lymphoma & $0 / 5(0 \%)$ & & & \\
\hline Mycosis fungoides & $0 / 5(0 \%)$ & & & \\
\hline Subcutaneous panniculitis-like T-cell lymphoma & $0 / 1(0 \%)$ & & & \\
\hline CD4-positive pleomorphic T-cell lymphoma & $0 / 1(0 \%)$ & & & \\
\hline T-cell large granular lymphocytic leukemia & $0 / 4(0 \%)$ & & & \\
\hline Overall & $14 / 200(7 \%)$ & & & \\
\hline
\end{tabular}

$P$-value between ALCL, ALK+, and ALCL, ALK-negative for cyclin D1 $(P=0.05)$, cyclin D2 $(P=0.28)$, cyclin D3 $(P=0.03)$, and pSTAT3 $(P=0.039)$.

Although cyclin D1 protein expression is well characterized in solid tumors and B-cell lymphomas, there are little data regarding cyclin D1 expression in peripheral T-cell lymphomas. ${ }^{7,8}$ In this retrospective study we evaluated the prevalence of cyclin D1 expression in peripheral T-cell lymphomas. The identification of cyclin D1 has treatment implications, because cyclin D1 can be a therapeutic target.

\section{Materials and methods}

Formalin-fixed, paraffin-embedded tissue from patients with a diagnosis of peripheral T-cell lymphoma was identified from the archives of three institutions (City of Hope National Medical Center, Mayo Clinic, and the University of Chicago). Institutional Review Board approval was obtained for this study at each center.

Four-micrometer-thick sections of formalin-fixed, paraffin-embedded tissue were cut from whole sections and tissue microarray blocks that were subsequently deparaffinized in xylene and hydrated using serial percentages of alcohol. Antibodies directed against cyclin D1 (clone SP4; 1:20 dilution, Thermo Fisher Scientific, Fremont, CA, USA), anticyclin D2 (clone aa1-30; 1:250 dilution, LSBio, Seattle, WA, USA), cyclin D3 (clone DCS22; 1:200 dilution, Cell Signaling Technology, Danvers, MA, USA), phospho-Stat3 (Tyr705) (M9C6, 1:50 dilution, Cell Signaling Technology), and SOX11 (clone MRQ-58; 1:100 dilution, Leica, Buffalo Grove, IL, USA) were used on these tissue sections. All stains were performed on the BOND immunostainer by Leica.

The above immunohistochemical stains were optimized on formalin-fixed, paraffin-embedded tissue with normal tonsils as the control. For all stains, a cutoff of $\geq 20 \%$ was used to assign as positive. Three pathologists (JYS, LS, and AF) independently scored all cases and any discrepant cases were resolved by re-scoring on a multi-headed microscope.

Fluorescence in situ hybridization was performed on formalin-fixed, paraffin-embedded tissue sections from whole tissue blocks and tissue microarrays using a previously reported method. ${ }^{9}$ Hybridization with the CCND1/IGH dual color, dual fusion probe (Abbott Molecular, Des Plaines, IL, USA) was used. Two hundred interphase nuclei were analyzed for each specimen analyzed. Two CCND1/IGH fusion signals (yellow) were scored as positive for CCND1/ IGH gene rearrangement.

\section{Results}

We identified 200 peripheral T-cell lymphomas from the three institutions and classified them using the World Health Organization Classification ${ }^{10}$. The cases included: 34 anaplastic large cell lymphoma, ALK-positive, 44 anaplastic large cell lymphoma, ALK-negative, 68 peripheral T-cell lymphomas, not otherwise specified, 24 angioimmunoblastic T-cell lymphomas, 7 extranodal NK/T-cell lymphomas, 4 enteropathy-associated T-cell lymphomas, 3 hepatosplenic T-cell lymphomas, 12 cutaneous T-cell lymphomas, and 4 large granular lymphocytic leukemias.

The clinical data on these cases were limited because many were referred only for hematopathology consultation or because clinical follow-up information was not available. Only two patients that were cyclin D1-positive had clinical follow-up and both had anaplastic large cell lymphoma, ALK+. One patient is in remission 2 years after initial treatment and the other patient went into remission after chemotherapy and autologous stem cell transplantation but relapsed 2 years later and subsequently underwent allogeneic stem cell transplantation, had relapsed disease, and was then treated with vorinostat and is in complete remission. 

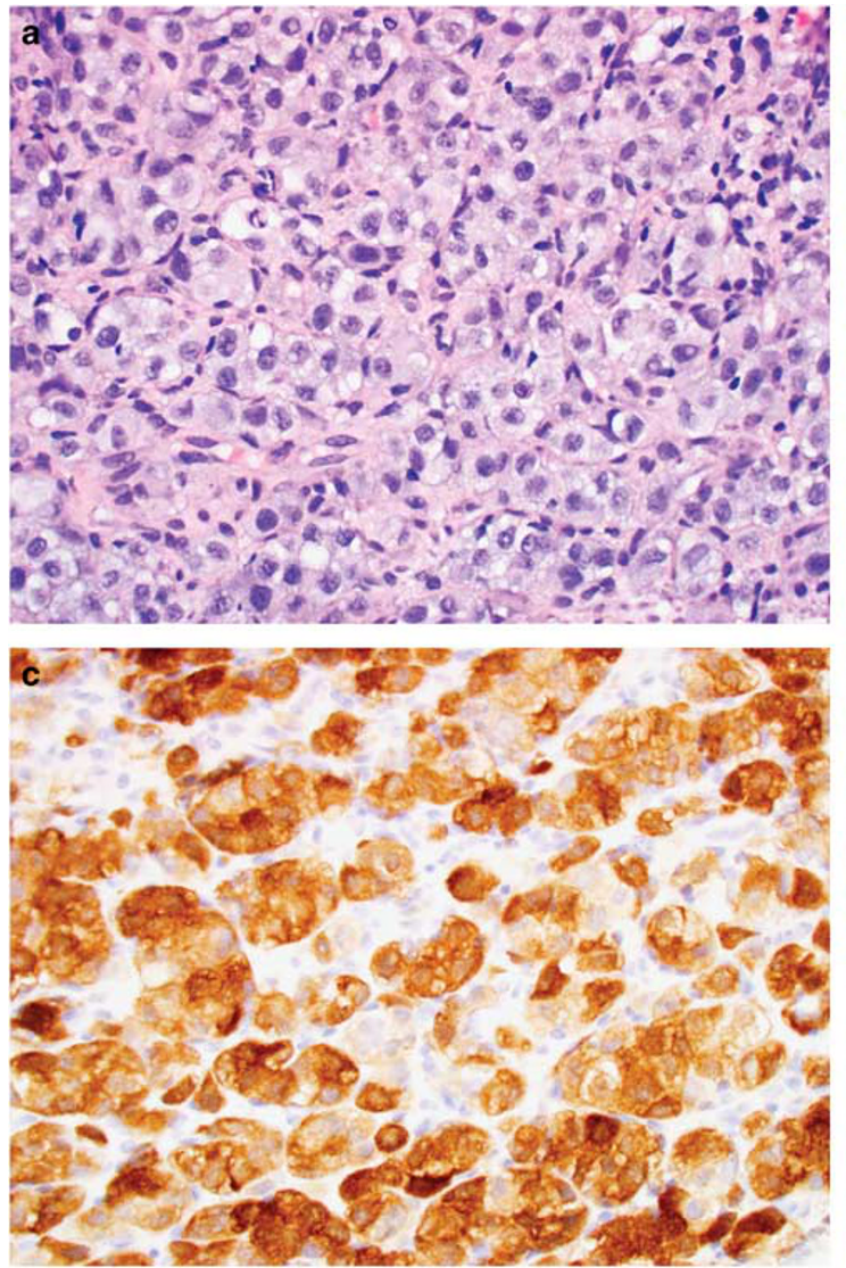
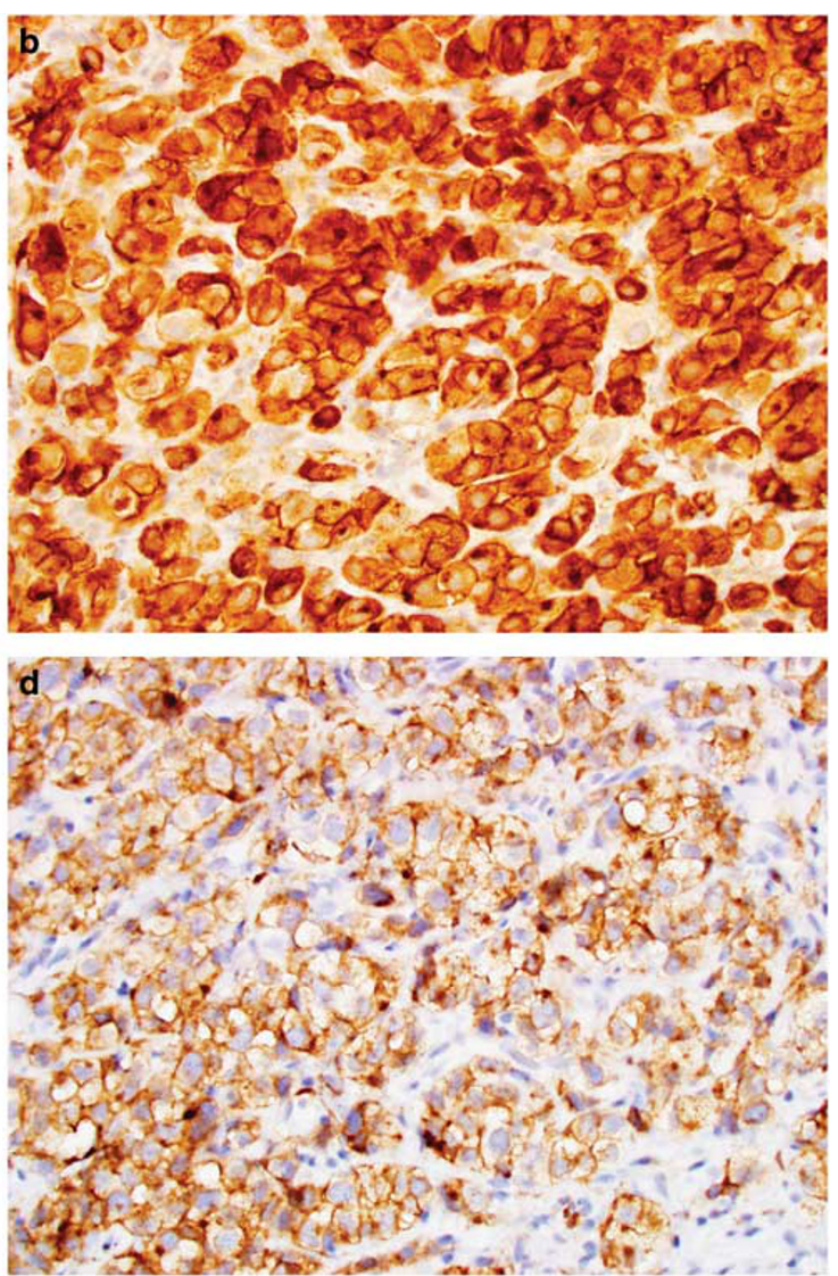
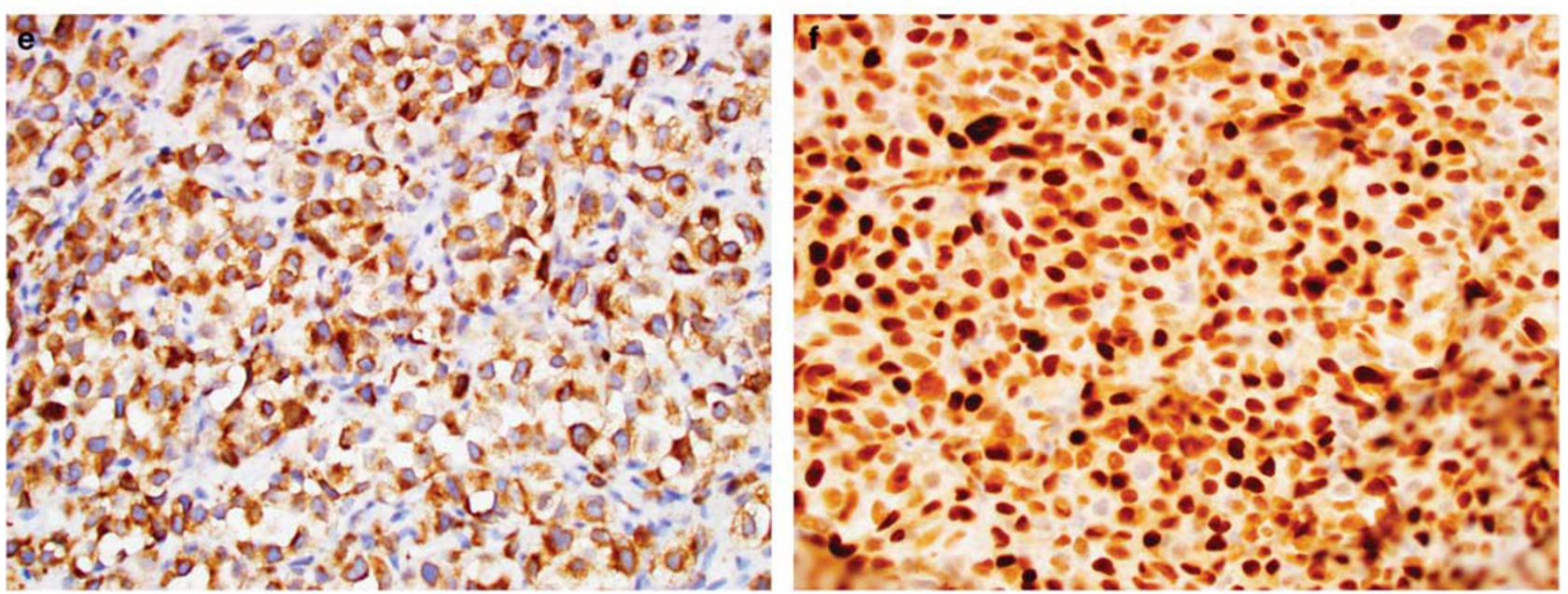

Figure 1 Anaplastic large cell lymphoma, ALK+. (a) H\&E stain showing pleomorphic cells in sheets. (b) These cells are strongly positive for CD30, (c) ALK protein (nuclear and cytoplasmic), (d) CD4, (e) perforin, and (f) cyclin D1 by immunohistochemistry.

Cyclin D1 staining was predominantly seen in anaplastic large cell lymphoma, ALK+ (8/34, 24\%) and anaplastic large cell lymphoma, ALK negative (3/44, $7 \%, P=0.05)$ (Table 1). Four of the anaplastic large cell lymphoma cases had diffuse nuclear staining (three anaplastic large cell lymphoma,
ALK+, one anaplastic large cell lymphoma, ALK - ) (Figure 1), whereas seven cases (five anaplastic large cell lymphoma, ALK+, two anaplastic large cell lymphoma, ALK - ) showed scattered nuclei positive but $>20 \%$ of the nuclei. The background endothelial cell nuclei were also positive and attention was 

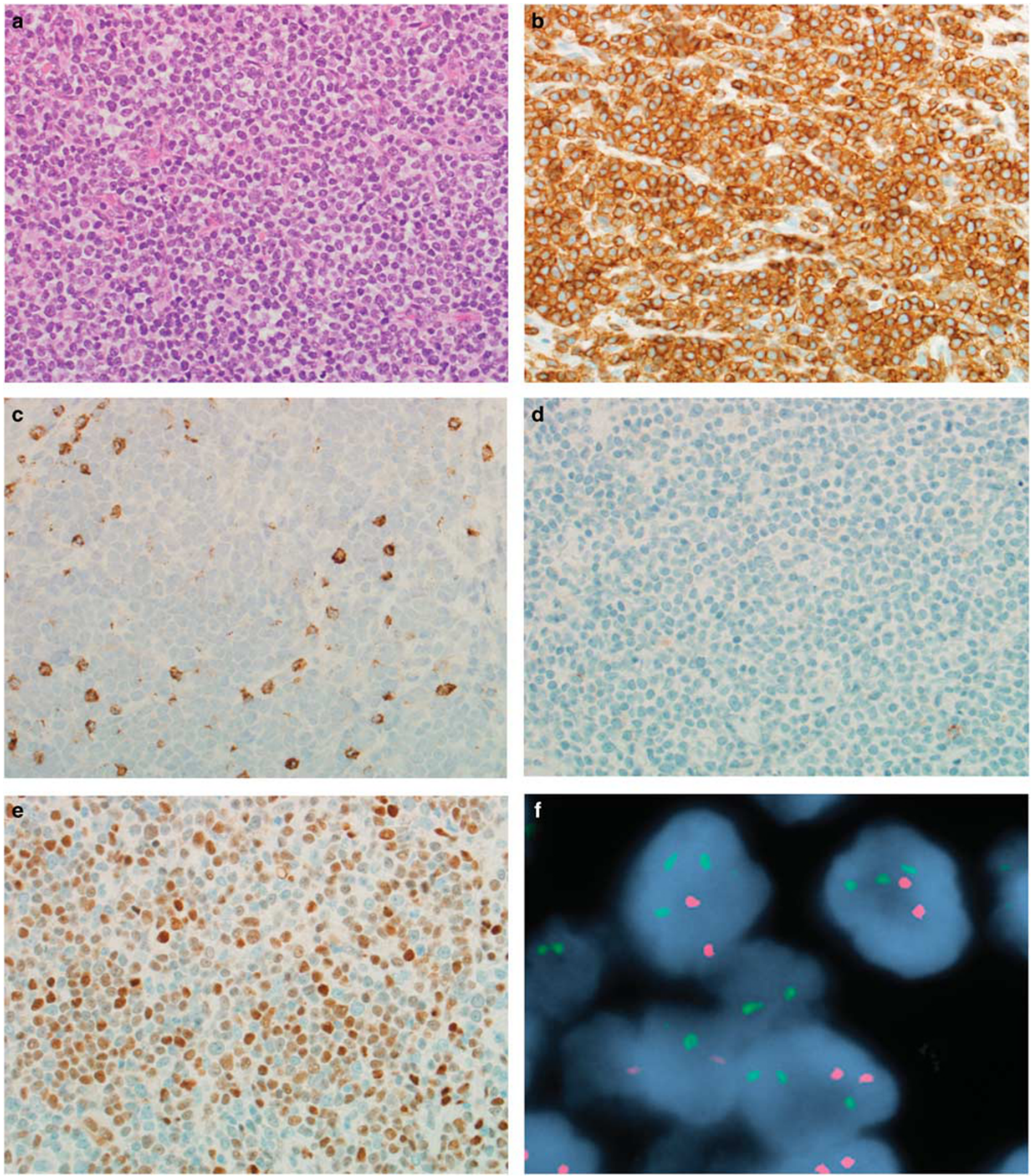

Figure 2 Peripheral T-cell lymphoma, not otherwise specified. (a) Sheets of mostly small tumor cells (H\&E). (b) The tumor cells are positive for CD3 and (c) show aberrant loss of CD2. (d) CD30 is negative. (e) Cyclin D1 is positive in many of the tumor cells. (f) Fluorescence in situ hybridization using a dual-fusion probe for CCND1 (red) and IGH (green) shows no evidence of IGH-CCND1 fusion or other abnormality of CCND1. There is an extra copy of the IGH locus. Fluorescence in situ hybridization image courtesy of Reid Meyer, Mayo Clinic, Rochester, MN.

taken not to confuse them with the neoplastic cells. Three of 68 cases $(4 \%)$ of peripheral T-cell lymphoma, not otherwise specified, were also positive (Figure 2); CD30 was negative in two of these cases and showed partial weak staining in one. All other peripheral T-cell lymphomas were negative for cyclin D1 protein expression. In the three diffusely cyclin D1-positive anaplastic large cell lymphoma, 

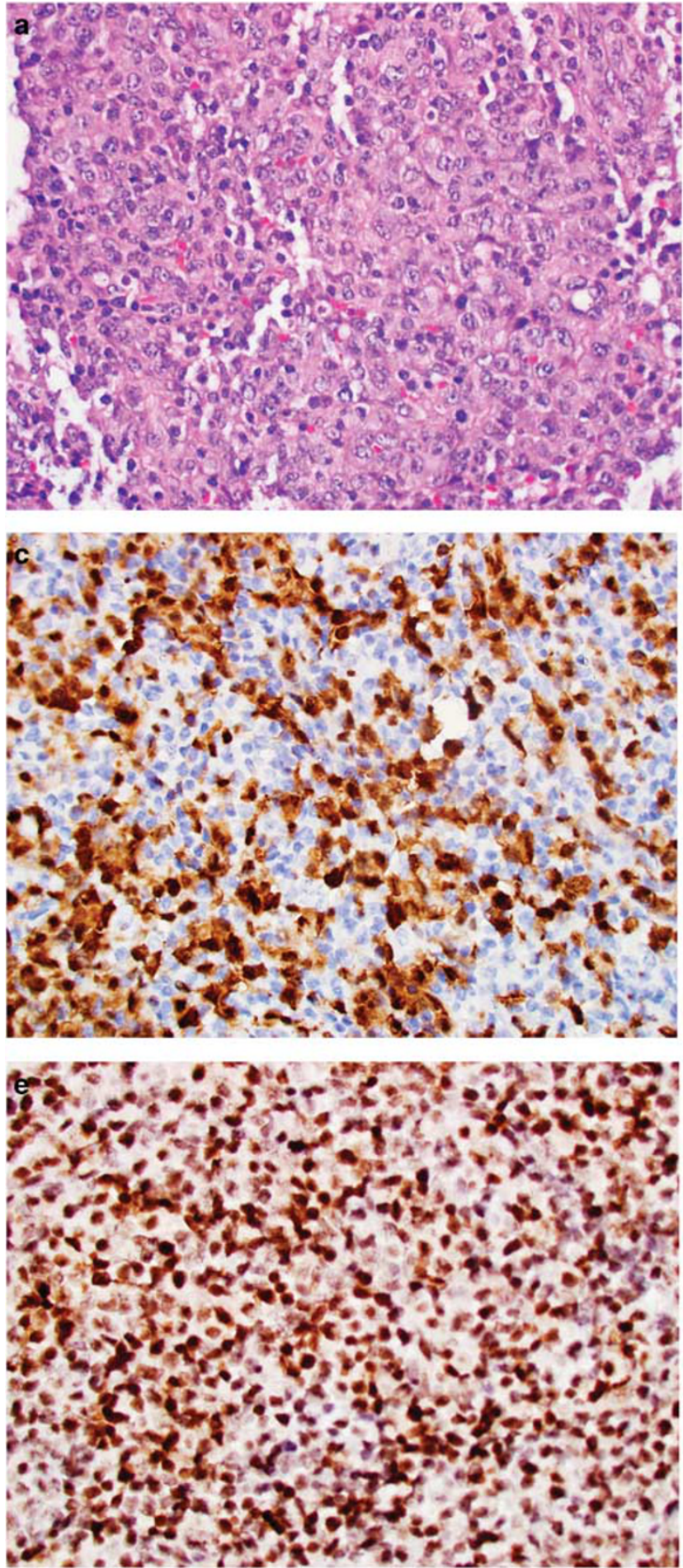
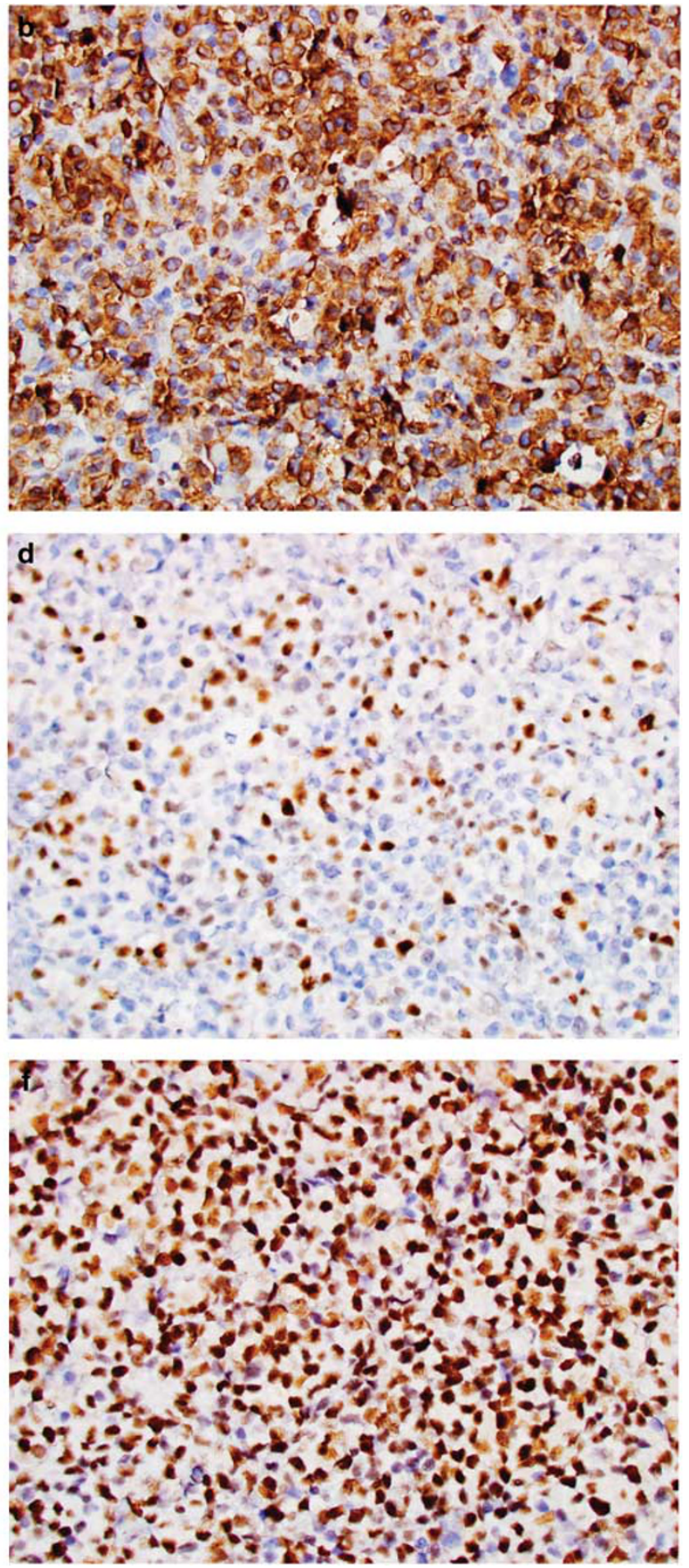

Figure 3 Anaplastic large cell lymphoma, ALK+. (a) Sheets of tumor cells with pleomorphic morphology (H\&E). (b) These cells are positive for CD30, (c) ALK1 protein, (d) focal cyclin D1, (e) cyclin D2, and (f) phospho-STAT3. The cells were also diffusely positive nuclear staining cyclin D3 (not shown).

ALK+ cases and one peripheral T-cell lymphoma, not otherwise specified case by immunohistochemistry, fluorescence in situ hybridization analysis was subsequently performed and was negative for $I G H / C C N D 1$ translocation as well as extra copies of the CCND1 gene (Figure 2e). SOX11 staining was also negative on all cyclin D1-positive cases by immunohistochemistry.

Owing to the higher incidence of cyclin D1 protein expression in anaplastic large cell lymphoma, 


\begin{tabular}{|l|l|l|l|l|l|l|l|l|l|l|l|l|l|l|l|l|l|l|l|l|l|l|l|l|l|l|l|l|l|}
\hline IHC & 1 & 2 & 3 & 4 & 5 & 6 & 7 & 14 & 15 & 16 & 17 & 18 & 19 & 8 & 9 & 10 & 11 & 12 & 13 & 20 & 21 & 22 & 23 & 24 & 25 & 26 & 27 & 28 & 29 \\
\hline ALK & & & & & & & & & & & & & & & & & & & & & & & & & & & & & \\
\hline pSTAT3 & & & & & & & & & & & & & & & & & & & & & & & & & & & & \\
\hline Cyclin D1 & & & & & & & & & & & & & & & & & & & & & & & & & & & & \\
\hline Cyclin D2 & & & & & & & & & & & & & & & & & & & & & & & & & & & & \\
\hline Cyclin D3 & & & & & & & & & & & & & & & & & & & & & & & & & & & & \\
\hline
\end{tabular}

Positive (>80\%)
Partial Positive (20-79\%)
Negative
Not performed

Figure 4 Immunohistochemical staining pattern of anaplastic large cell lymphoma. IHC, immunohistochemistry.

additional immunohistochemical stains were performed on this group that had available additional material to investigate the JAK/STAT pathway. The majority of anaplastic large cell lymphoma, ALK+ cases were positive for cyclin D2 (10/12, 83\%) and cyclin D3 (12/12, 100\%). As for anaplastic large cell lymphoma, ALK-negative cases, cyclin D2 (7/15, $47 \%, P=0.28)$, and cyclin D3 $(5 / 15,33 \%, P=0.03)$ were less frequently positive. As expected, phosphoSTAT3 (pSTAT3) was expressed in more cases of anaplastic large cell lymphoma, ALK+ (13/13, 100\%) compared with ALK-negative cases (6/16, 38\%, $P=0.039$ ) (Figures 3 and 4).

\section{Discussion}

Cyclin D1 protein expression is commonly seen in B-cell neoplasms, classically in mantle cell lymphoma but also in hairy cell leukemia and plasma cell myeloma. In this study, we show that the expression of cyclin D1 is not limited to B-cell lymphomas but can also be seen in some peripheral T-cell lymphomas, particularly anaplastic large cell lymphoma and is not associated with translocation or extra copies of the CCND1 gene.

In our study, cyclin D1 protein expression was highest in the anaplastic large cell lymphoma, ALK+ cases (24\%) compared with ALK - (7\%) and peripheral T-cell lymphoma, not otherwise specified (4\%). Normally, the cell cycle is tightly regulated by molecules such as the cyclins, cyclin-dependent kinases, and cyclin-dependent kinase inhibitors. Cyclin D1 overexpression induces G1 phase progression by activating cyclin-dependent kinases, which phosphorylates substrates such as RB and other transcription factors that are important in proliferation and differentiation. ${ }^{3,4,11-13}$ In anaplastic large cell lymphoma, ALK+, the NPM-ALK fusion protein binds to and phosphorylates STAT3, which results in constitutive activation. ${ }^{14-16}$ STAT3 targets many genes including the anti-apoptotic proteins (eg, Bcl-xL and Bcl-2), as well as proliferation-associated proteins such as myc and cyclin D1. ${ }^{17,18}$ Constitutively activated STAT3 has been shown to promote tumorigenesis and has been found to increase cyclin D1 and myc mRNA levels approximately 3-5 fold in transformed cells. ${ }^{18}$

Phospho-STAT3 was more commonly expressed in anaplastic large cell lymphoma, ALK+ $(100 \%)$ as compared with ALK - $(38 \%, P=0.039)$. Interestingly, in the anaplastic large cell lymphoma cases that were ALK+ and expressed pSTAT3, most of these cases expressed two or more of the cyclin D proteins $(83 \%)$, whereas only two $(13 \%)$ of the ALK - and pSTAT3-positive cases expressed any cyclin D proteins. Cyclin D3 expression in anaplastic large cell lymphomas has been described before and our findings are consistent with the notion that activation of STAT3 in ALK-positive cases leads to the overexpression of the cyclin proteins promoting cell proliferation. ${ }^{19}$

Prior studies investigating cyclin D1 expression in peripheral T-cell lymphoma reported negative findings, likely due to the limited number of cases evaluated (eg, only 3-7 cases of anaplastic large cell lymphoma, ALK+). ${ }^{20,21}$ Only a few cases of anaplastic large cell lymphoma, ALK+, showed diffuse nuclear staining for cyclin D1 protein, whereas other cases showed only focal staining in the neoplastic cells. Many of the negative cases showed positivity in the background endothelial cells. Unfortunately, clinical follow-up on the majority of our cyclin D1-positive T-cell lymphomas was unavailable and, therefore, it is unclear whether cyclin D1 protein expression impacts prognosis.

Although cyclin D1 overexpression is not specific to any particular cancer, it is important in oncogenesis and proliferation of many tumors such as squamous cell carcinomas of the head and neck, ${ }^{22}$ breast cancers, ${ }^{23}$ and colorectal carcinomas. ${ }^{24}$ Cyclin D1 has been frequently associated with some specific subtypes of B-cell lymphomas but, as our study shows, can also been in peripheral T-cell lymphomas. The overexpression of cyclin $\mathrm{D}$ proteins is seen preferentially in anaplastic large cell lymphoma, ALK+, most likely due to activation of the JAK/STAT pathway. ${ }^{25}$ Routine staining for cyclin D1 protein in peripheral T-cell lymphomas will identify patients who may benefit from therapies that target cyclin D1 or its associated cyclin-dependent kinases. ${ }^{13,26}$ 


\section{Disclosure/conflict of interest}

The authors declare no conflict of interest.

\section{References}

1 Kim JK, Diehl JA. Nuclear cyclin D1: an oncogenic driver in human cancer. J Cell Physiol 2009;220: 292-296.

2 Albanese C, Johnson J, Watanabe G et al. Transforming p21ras mutants and c-Ets-2 activate the cyclin D1 promoter through distinguishable regions. J Biol Chem 1995;270:23589-23597.

3 Cheng M, Sexl V, Sherr CJ et al. Assembly of cyclin D-dependent kinase and titration of p27Kip1 regulated by mitogen-activated protein kinase kinase (MEK1). Proc Natl Acad Sci USA 1998;95:1091-1096.

4 Hanahan D, Weinberg RA. Hallmarks of cancer: the next generation. Cell 2011;144:646-674.

5 Bertoni F, Rinaldi A, Zucca E et al. Update on the molecular biology of mantle cell lymphoma. Hematol Oncol 2006;24:22-27.

6 Chesi M, Bergsagel P, Brents L et al. Dysregulation of cyclin D1 by translocation into an IgH gamma switch region in two multiple myeloma cell lines [see comments]. Blood 1996;88:674-681.

7 Yatabe Y, Suzuki R, Matsuno Y et al. Morphological spectrum of cyclin D1-positive mantle cell lymphoma: Study of 168 cases. Pathol Int 2001;51:747-761.

8 Warnke RA, Jones D, ED Hsi. Morphologic and immunophenotypic variants of nodal T-cell lymphomas and T-cell lymphoma mimics. American Journal of Clinical Pathology 2007;127:511-527.

9 Pullarkat V, Bedell V, Kim Y et al. Neoplastic mast cells in systemic mastocytosis associated with $\mathrm{t}(8 ; 21)$ acute myeloid leukemia are derived from the leukemic clone. Leuk Res 2007;31:261-265.

10 Swerdlow S, Campo E, Harris NL et al. In: WHO Classification of Tumours of Haematopoietic and Lymphoid Tissues. International Agency for Research on Cancer: Lyon, France, 2008, pp 270-319.

11 Hanahan D, Weinberg RA. The hallmarks of cancer. Cell 2000;100:57-70.

12 Lovec H, Sewing A, Lucibello FC et al. Oncogenic activity of cyclin D1 revealed through cooperation with Ha-ras: link between cell cycle control and malignant transformation. Oncogene 1994;9:323-326.
13 Musgrove EA, Caldon CE, Barraclough J et al. Cyclin D as a therapeutic target in cancer. Nat Rev Cancer 2011; 11:558-572.

14 Zamo A, Chiarle R, Piva R et al. Anaplastic lymphoma kinase (ALK) activates Stat3 and protects hematopoietic cells from cell death. Oncogene 2002;21:1038-1047.

15 Zhang Q, Raghunath PN, Xue L et al. Multilevel dysregulation of STAT3 activation in anaplastic lymphoma kinase-positive T/null-cell lymphoma. J Immunol 2002;168:466-474.

16 Khoury JD, Medeiros LJ, Rassidakis GZ et al. Differential expression and clinical significance of tyrosinephosphorylated STAT3 in ALK+ and ALK- anaplastic large cell lymphoma. Clin Cancer Res 2003;9: 3692-3699.

17 Bromberg J. Stat proteins and oncogenesis. J Clin Invest 2002;109:1139-1142.

18 Bromberg JF, Wrzeszczynska MH, Devgan G et al. Stat3 as an oncogene. Cell 1999;98:295-303.

19 Dalton RR, Rassidakis GZ, Atwell C et al. Differential expression of cyclin D3 in ALK+ and ALK- anaplastic large cell lymphoma. Hum Pathol 2005;36:806-811.

20 Metcalf RA, Zhao S, Anderson MW et al. Characterization of D-cyclin proteins in hematolymphoid neoplasms: lack of specificity of cyclin-D2 and D3 expression in lymphoma subtypes. Mod Pathol 2010;23:420-433.

21 Elenitoba-Johnson KS, Bohling SD, Jenson SD et al. Fluorescence PCR quantification of cyclin D1 expression. J Mol Diagn 2002;4:90-96.

22 Nakagawa H, Zukerberg L, Togawa K et al. Human cyclin D1 oncogene and esophageal squamous cell carcinoma. Cancer 1995;76:541-549.

23 Zukerberg LR, Yang WI, Gadd M et al. Cyclin D1 (PRAD1) protein expression in breast cancer: approximately one-third of infiltrating mammary carcinomas show overexpression of the cyclin D1 oncogene. Mod Pathol 1995;8:560-567.

24 Bartkova J, Lukas J, Strauss M et al. The PRAD-1/cyclin D1 oncogene product accumulates aberrantly in a subset of colorectal carcinomas. Int J Cancer 1994;58: $568-573$.

25 Matsui T, Kinoshita T, Hirano T et al. STAT3 downregulates the expression of cyclin $\mathrm{D}$ during liver development. J Biol Chem 2002;277:36167-36173.

26 Sausville EA, Zaharevitz D, Gussio R et al. Cyclindependent kinases: initial approaches to exploit a novel therapeutic target. Pharmacol Ther 1999;82: 285-292. 